\title{
Clinical Trial Regulations In India : Past, Present and Future
}

\author{
Haroon Rashid, M.Sc \\ Clinical Research Assistant, Department of Pharmacology, AllMS, New Delhi
}

\section{INTRODUCTION}

Clinical trials are the only way of establishing the safety and efficacy of any new drug before its introduction in the market for human use. Clinical trials (with safeguards) are necessary for introduction of new drugs for a country like India, considering its disease burden and emergence of new variants of disease.

The regulatory bodies need to frame guidelines and regulatory approval processes on a par with international standards. Many of the new laws, guidance documents, notifications and initiatives for regulating pharmaceutical industry were in the charts for quite a long time. Indian regulatory authorities have started looking into speedy implementation and providing support in terms of necessary infrastructure and investment.

Central Drugs Standard Control Organization (CDSCO) which comes under the Ministry of Health and Family Welfare is the main body which works on development of regulatory procedures and standards for drugs, cosmetics, diagnostics and devices. It lays down regulatory guidance by amending acts and rules; and regulates new drug approval process. Its main objective is to standardize clinical research and bring safer drugs to the Indian market. The Drug Controller General of India DCGI is responsible for giving regulatory permissions for the conduct of clinical trials and is responsible for approval of marketing licenses for drugs in India.

Along with the DCGI office, there are other

\author{
Correspondence \\ Haroon Rashid, \\ Clinical Research Assistant, \\ Department of Pharmacology, AlIMS, New Delhi
}

governmental bodies involved in the pharmaceutical regulations of the new drugs. When an application is filed, the office of the DCGI reviews it; the required time for approval depends on the trial's regulatory status in other countries. Along with the application submitted to the DCGI office, parallel submission can be made to the ethics committees of would-be sites, which are usually hospitals and/or clinics. These Ethics committees are usually affiliated with study sites; or sometimes they function as independent ethics committees. The standard time required for ethics committee approval is 4 to 6 weeks. Many study sites have scientific review committees (SRC) to review the scientific rationale of the study. Once the study is passed through and approved by the SRC then it is submitted to the ethics committee for their assessment.

Status of the new drug would define the procedure for applying for marketing approval. It can be largely classified into following categories:

a) New drug substances discovered that are already approved/marketed in other countries;

b) New drug substances discovered that are not approved/marketed in other countries;

c) New drug substances discovered in India.

In case of the (a) category, it is sufficient if Phase III (therapeutic confirmatory) trials are conducted in a large number of patients with multiple site and compared with a standard drug, to confirm efficacy and safety. For the category (b), permission for clinical trials is given with a "phase lag". For example, phase I study for a new drug is allowed only if the drug has completed phase $\mathrm{I}$ in other country and moved to the next phase. In the case of new drug substances discovered in India i.e. category (c), Phase I clinical trials need to be carried out in India. However, this 
has been recently revised, and now in the case of new drug substances not yet approved or marketed in other countries, Schedule Y now allows clinical trials to be carried out in India, concurrently with the trials abroad.

\begin{tabular}{|c|c|}
\hline \multicolumn{2}{|c|}{$\begin{array}{l}\text { REGULATORY BODIES IN INDIA } \\
\text { INVOLVED IN DRUG REGULATION }\end{array}$} \\
\hline Central Drugs & Regulatory body \\
\hline Standard Control & controlled and \\
\hline Organization & governed by \\
\hline$(\mathrm{CDSCO})$ & $\begin{array}{l}\text { Directorate General } \\
\text { of Health Services of } \\
\text { Ministry of Health } \\
\text { and Family Welfare } \\
\text { which regulates } \\
\text { drugs in India }\end{array}$ \\
\hline Drugs Controller & Regulatory agency \\
\hline $\begin{array}{l}\text { General India } \\
\text { (DCGI) }\end{array}$ & $\begin{array}{l}\text { under the } \\
\text { government of India }\end{array}$ \\
\hline & $\begin{array}{l}\text { whose function is to } \\
\text { assess the quality, } \\
\text { safety and efficacy } \\
\text { of a drug }\end{array}$ \\
\hline Indian Council of & Apex body in India \\
\hline $\begin{array}{l}\text { Medical Research } \\
\text { (ICMR) }\end{array}$ & $\begin{array}{l}\text { that formulates, } \\
\text { co-ordinates and }\end{array}$ \\
\hline & $\begin{array}{l}\text { promotes biomedical } \\
\text { research }\end{array}$ \\
\hline Drugs & Provides technical \\
\hline Consultative & guidance to the \\
\hline Committee (DCC) & Central Drugs \\
\hline & Standard Control \\
\hline & Organization \\
\hline Central Drugs & National statutory \\
\hline Laboratory (CDL) & $\begin{array}{l}\text { laboratory of the } \\
\text { Indian government } \\
\text { for quality control of } \\
\text { drugs }\end{array}$ \\
\hline Drugs Technical & Provides technical \\
\hline $\begin{array}{l}\text { Advisory Board } \\
\text { (DTAB) }\end{array}$ & $\begin{array}{l}\text { guidance to the } \\
\text { CDSCO }\end{array}$ \\
\hline & \\
\hline
\end{tabular}

The Drugs \& Cosmetics Act, 1940 contains enabling provisions for regulating and ensuring quality, safety and efficacy of drugs and, therefore, contains inherent enabling powers for regulating the clinical trials. Accordingly, under the inherent plenary powers vested in the Act, necessary rules, procedures and guidelines have been framed under the Drugs and Cosmetics Rules, 1945. Regulatory provisions for conducting clinical trials in the country are prescribed under Rule-122DA, 122DAA, 122DAB, 122DAC, 122DD, 122E and Schedule $Y$ to the Drug and Cosmetics Rules, 1945. CURRENT SCENARIO OF CLINICAL TRIALS IN INDIA

The Clinical Trials on new Drugs are regulated under the provisions of Drug and Cosmetics Rules 1945 as amended from time to time. The Drugs and Cosmetics Rules provides that no Clinical Trial for a new Drug, whether for Clinical investigations or any Clinical experiment by any institution, shall be conducted except under and in accordance with the Permission in writing of the Licensing authority defined in clause(b) of rule 21 i.e. Drug Controller General of India. The detailed requirements and guidelines for undertaking Clinical trials are provided under Schedule $\mathrm{Y}$ to the said rule. Schedule $\mathrm{Y}$ further specify that the Clinical trial is conducted and data generated, documented and reported in compliance with the protocol and Good Clinical Practice (GCP) guidelines specified for the purpose as well as with all applicable statutory provisions. Clinical trials are conducted in compliance to the provisions of the Drug and Cosmetic rules and the care is taken to protect the rights, safety, and wellbeing of the subjects participating in the Trial and data generated is scientifically and ethically Sound. (F.No. DCGI/MISC/2013(107))

The Schedule Y of Drugs and Cosmetics Rules specifies that it is the responsibility of Ethics committee that reviews and accords its approval to a trial protocol to safeguard the Rights, Safety and wellbeing of all Trial subjects. The clause 3.1.7 of the GCP guidelines under Para "Confirmation of Review by the Ethics Committee" requires that Sponsor shall obtain from the Investigator(s) and/or institutions, an undertaking that the Ethics Committees organized and operated according to the GCP guidelines and the applicable regulations. Any activities in Clinical trial in contravention to any provision of the Schedule Y, the GCP guidelines or other applicable regulations will be viewed as noncompliance to the statutory requirements and action will be taken as per the Drugs and Cosmetics Act and Rules. (F.No. DCGI/MISC/2012)

In all trials, a freely given, informed, written consent is required to be obtained from each study subject. The 


\begin{tabular}{ll} 
Important forms of Drug and cosmetic Act for clinical research \\
\hline FORM 44 & $\begin{array}{l}\text { Application for grant of permission to import or manufacture a } \\
\text { New Drug or to undertake clinical trial }\end{array}$ \\
FORM 12 & $\begin{array}{l}\text { Application for license to import drugs for the purposes of } \\
\text { examination, test or analysis } \\
\text { License to import drugs for the purposes of examination, test or } \\
\text { analysis (Validity is one year) }\end{array}$ \\
FORM 11 & $\begin{array}{l}\text { Issue of import certificate (Validity is six months) } \\
\text { Application form for issue of import certificate for } \\
\text { FOrcotic drugs and psychotropic substance } 04\end{array}$ import of \\
FORM 01 &
\end{tabular}

\begin{tabular}{|c|c|}
\hline \multicolumn{2}{|c|}{ Important Rules of Drug and cosmetic Act for clinical research } \\
\hline Rule 34 & Application for license for examination, test or analysis \\
\hline Rule 122-A & Application for permission to import new drug \\
\hline Rule 122-D & Permission to import fixed dose combination \\
\hline Rule 122-DA & $\begin{array}{l}\text { Application for permission to conduct clinical trials for new } \\
\text { drug/investigational new drug }\end{array}$ \\
\hline Rule 122-DAA & Definition of clinical trial \\
\hline Rule $122-E$ & Definition of new drug \\
\hline Rule 55 & Application for import certificate \\
\hline Rule 56 & Issue of Import certificate \\
\hline NEW RULES & (D \& C Act Amendment 2013) \\
\hline Rule 122 DAB & Compensation in case of injury or death during Clinical Trial \\
\hline Rule 122 DAC & Permission to conduct to Clinical Trials \\
\hline Rule 122 DD & Registration of Ethics committee. \\
\hline
\end{tabular}

Investigator must provide information about the study verbally as well as using a patient information sheet, in a language that is non-technical and understandable by the study subject. The Subject's Consent must be obtained in writing using an 'Informed Consent Form'. Both the Patient information sheet and Informed consent Form should have been approved by the Ethics committee and furnished to licensing authority. Any changes in these documents should be approved by the Ethics committee and submitted to the licensing authority before such changes are implemented. An essential element to be included in the study's Informed Consent document as well as format for the Informed Consent Form for study subjects as per Appendix V of Schedule Y of Drugs and Cosmetic rules. (F.No.12-01/12DC (Pt-77))

The Clinical trial can be initiated only:

(i) After permission from the Drugs Controller General (India) [DCGI],

(ii) Approval from respective Ethics Committee and

(iii)Mandatory registration on website www.ctri.in being maintained by ICMR before the enrolment of the first trial participant for the clinical trial.

\section{Changes made in the Regulatory System}

A number of significant changes In the Regulatory system that have been made will only serve to make clinical trials in India slow and expensive, compelling sponsors to look at alternatives. These changes include:

- Rules on compensation for clinical trial-related injury or death

- Expert committee to advise CDSCO (the drug regulator's office) on compensation

- Formula to determine the quantum of compensation in the cases of serious adverse events of deaths occurring during clinical trials

- Registration of ethics committees

- Apex committee and Technical committee to supervise and monitor clinical trials

- Expert committee to formulate policy, guidelines, SOPs for approval of new drugs, clinical trials and banning of drugs

- Audio-video recording of informed consent process

- Regulatory inspections for checking compliance to good clinical practices 
- Process for clinical trial approval

- Furnishing of information regarding financial support to investigators

- The Drugs And Cosmetics (Amendment) Bill, 2013 (Future of Regulatory system)

Measures taken to strengthen the regulation of clinical trials

A. Regulatory Measures:

i. 12 New Drug Advisory Committees (NDAC) and 6 Medical Device Advisory Committees have been constituted. Fresh Applications of Clinical Trial proposals of new drug substances except Investigational New Drug (INDs) are being evaluated by these Committees.

ii. All IND applications are evaluated by IND committees.

iii. Registration of Clinical Trial in ICMR registry has been made mandatory.

iv. Guidelines for conducting Clinical Trial inspection of site and sponsor / CROs have been prepared and Posted on CDSCO website.

To ensure transparency in approval of proposals for conduct of clinical trials for drugs by the DCGI, 12 New Drug Advisory Committees (NDAC) consisting of experts from the government medical colleges and eminent institutions from all over the country have been constituted. Fresh applications of clinical trial proposals of new drug substances excluding investigational new drugs (INDs) are being evaluated by these Committees. For INDs, two separate expert committees have similarly been constituted. Similarly, for medical devices, 6 Medical Device Advisory Committees (MDAC) have been constituted.

- 12 New Drug Advisory Committees (NDAC) each with 10 experts. These include:

- Reproductive and Urology

- Cardiovascular and Renal

- Ophthalmology

- Dermatology and Allergy

- Analgesics, Anaesthetics and Rheumatology

- Pulmonary

- Gastroenterology and Hepatology

- Metabolism and Endocrinology

- Neurology and Psychiatry
- Oncology and Haematology

- Anti-microbial, Anti-parasitic, Anti-fungal and Antiviral

- Vaccines

- 6 Medical Device Advisory Committees (MDAC) include

- Cardiovascular

- Orthopaedic

- Ophthalmic

- Reproductive and Urology

- Dental

- General Experts pool

Each committee has 5 experts except the General Experts Pool that has 12 experts.

\section{A. Amendments made in Drug and Cosmetic Rules to strengthen Regulation of Clinical Trial:}

I. Amendment vide G.S.R 53(E) dated 30-01-2013 specifying procedures to analyse reports of Serious Adverse Events (SAEs) occurring during clinical trial and procedures for payment of compensation in case of trial related injury or death as per prescribed timelines.

II. Amendment vide G.S.R 63(E) dated 01-02-2013 specifying various conditions for conduct of clinical trials, authority for conduct of clinical trial inspections and actions in case of non-compliance.

III. Amendment vide G.S.R 72(E) dated 08-02-2013 specifying requirements and Guidelines for Registration of Ethics committee.

Clinical Trials in India (30.8.2013) by Ministry of Health \& Family Welfare

\section{SAE reporting and compensation:}

Amendment vide Gazette Notification G.S.R. 53(E) dated 30-01-2013 specifying procedures to analyse the reports of Serious Adverse Events occurring during clinical trials and procedures for payment of compensation in case of trial related injury or death as per prescribed timelines as under:

I. Insertion of a new Rule $122 \mathrm{DAB}$ relating to compensation in case of injury or death during clinical trials.

a) Free medical management in case of injury.

b) Financial compensation in case of injury or death due to: 
i. Adverse effect of investigational product.

ii. Violation of the approved protocol, scientific misconduct or negligence by the sponsor or his representative or the investigator;

iii. Failure of investigational product to provide intended therapeutic effect;

iv. Use of placebo in a placebo-controlled trial;

v. Adverse effects due to concomitant medication excluding standard care.

vi. For injury to a child in utero.

vii. Any clinical trial procedures involved in the study.

II. Expansion of responsibilities of Sponsor, Investigator and Ethics Committees to ensure that the report of Serious Adverse Events (SAEs) including deaths are reported, analysed within the prescribed timelines and in case of clinical trial related injury or death, compensation are paid as per the prescribed procedures.

Insertion of definition of SAEs and detailed procedures for reporting and examination of such events in Schedule 'Y'.

III. The check list for trial participants' informed consent documents have been amended to include statements describing that in the event of injury of the trial participant, he/she shall be provided free medical management as long as required and in the event of clinical trial related injury or death, the Sponsor or his representative shall provide financial compensation for the injury or death.

IV. The Format of Informed Consent Form for clinical trial participants have been amended to capture the information relating to address, qualification, occupation, annual income of the trial participant and name and address of his nominee (for the purpose of compensation in case of trial related death). It has also been made mandatory for the investigator to hand over a copy of the patient information sheet and duly filled Informed Consent Form to the trial participant or his/her attendant.

V. Insertion of a separate Appendix XII related to compensation in case of injury or death during clinical trials in Schedule ' $\mathrm{Y}$ '. The Appendix prescribes the detailed procedures for examination of SAE reports including deaths and payment of financial compensation in case of trial related injury or death as per the prescribed timelines.

As per the procedures:-

a) Investigator shall report all SAEs to the DCGI, Sponsor or his representative and the Ethics Committee within 24 hours of their occurrence.

b) In case of death, an independent Expert Committee constituted by DCG (I) shall examine the case and give recommendations to DCG (I) to determine the cause of death and also to decide the quantum of compensation, in case of clinical trial related death. The independent Expert Committee, while examining the event, may take into consideration the reports of the Investigator, the Sponsor or his representative and the Ethics Committee. The DCG (I), after considering the recommendations of the Expert Committee, shall determine the cause of death and decide on the quantum of compensation to be paid by the sponsor or his representative in case of trial related deaths within three months of receiving the report of SAE of death.

c) In case of SAEs other than death, the DCG (I), after considering the reports of the Investigator, the Sponsor and the Ethics Committee, shall determine the cause of injury and also decide the quantum of compensation to be paid by the Sponsor or his representative in case of clinical trial related injury within three months of receiving the report. The DCG(I) will have the option to constitute an Expert Committee to examine such SAEs and recommend the quantum of compensation to be paid, if any.

Clinical Trials in India (30.8.2013) by Ministry of health \& family welfare

Formula To Determine The Quantum Of Compensation In The Cases Of Clinical Trial Related Serious Adverse Events (Saes) Of Deaths Occurring During Clinical Trials (http://www.cdsco.nic.in/)

The Drugs and Cosmetics Rules have been amended vide GSR 53(E) dated 30-01-2013 inserting a Rule $122 \mathrm{DAB}$ and a new Appendix-XII in Schedule ,Y $\mathrm{Y}^{\mathrm{ee}}$. The amendment specifies the procedure for processing of reports of Serious Adverse Events (SAEs) including deaths occurring during clinical trial to arrive at the cause of death/injury to the subject, and to determine the quantum of compensation, if any, to be paid by the Sponsor or his representative, whosoever have obtained permission from the $\mathrm{DCG}(\mathrm{I})$ in a time bound manner. 
the $\mathrm{DCG}(\mathrm{I})$ in a time bound manner.

As per the provisions of the amendment, an Independent Expert Committee shall examine the report of serious adverse event of death and give its recommendation to the Licensing Authority within 30 days of receiving the report from the concerned Ethics Committee. The DCG(I) shall, then decide the Quantum of Compensation to be paid by the Sponsor or his representative and shall pass order as deemed necessary within three months of receiving the report on the Serious Adverse Event of death.

In case of clinical trial related injury or death, the Sponsor or his representative shall pay the compensation as per the order of the DCG(I) within thirty days of the receipt of such order.

Criteria and formula for determining the quantum of compensation in case of clinical trial related deaths

(http://www.cdsco.nic.in/)

COMPENSATION $=$ B $\quad \begin{array}{r}\text { FxR } \\ 99.37\end{array}$

Where,

$\mathrm{B}=$ Base amount (i.e. 8 lacs)

$\mathrm{F}=$ Factor depending on the age of the subject as per (based on Workmen Compensation Act)

$\mathrm{R}=$ Risk Factor depending on the seriousness and severity of the disease, presence of co-morbidity and duration of disease of the subject at the time of enrolment in the clinical trial between a scale of 0.5 to 4 .

Thus, it will be seen that the compensation amount will vary from a minimum of Rs. 4 lacs to a maximum of Rs. 73.60 lacs depending on the age of the deceased and the risk factor. However, in case of patients whose expected mortality is $90 \%$ or more within 30 days, a fixed amount of Rs. 2 lac should be given.

\section{Permission to conduct clinical trials}

Amendment vide Gazette Notification G.S.R. 63(E) dated 01-02-2013 specifying various conditions for conduct of clinical trials, authority for conducting clinical trial inspections and actions in case of non-compliance, as under:

A new Rule 122DAC has been inserted, the salient features of which are as under:

i. Clinical trial shall be conducted in compliance with the approved protocols, requirements of Schedule Y, Good Clinical Practices (GCP) Guidelines for conduct of clinical trials in India and other applicable regulations;

ii. Approval of the Ethics Committee shall be obtained before initiation for the study;

iii. Clinical trial shall be registered at Clinical Trials Registry of India before enrolling the first patient for the study;

iv. The Sponsor and his representative and clinical trial sites and the Investigator shall allow officers authorized by the Central Drugs Standard Control Organisation (CDSCO), who may be accompanied by an officer of the State Drug Control Authority concerned, to enter with or without prior notice into their premises and clinical trial sites to inspect, search and seize any record, data, document, books, investigational drugs, etc.

v. In case of non-compliance to the provisions of clinical trials by any Sponsor including the representative, investigators conducting clinical trial and clinical trial sites, DCG (I) can take following actions:

a) Reject or discontinue the study.

b) Suspend or cancel the clinical trial permission;

c) Debar the Investigator(s), Sponsor including his representative to conduct any clinical trial in future.

Clinical Trials in India $(30.8$.2013) by Ministry of health \& family welfare

\section{Registration and functioning of ethics committee:}

Amendments vide Gazette Notification G.S.R No. 72(E) dated 08-02-2013 specifying requirements and guidelines for registration of Ethics Committee, as under:

As per Rule 122DD:-

i. No Ethics Committee shall review and accord its approval to a clinical trial protocol without prior registration with $\mathrm{DCG}(\mathrm{I})$.

ii. An application for registration of Ethics Committee is required to be made to DCG (I) along with detailed information about the committee as per Appendix VIII of Schedule Y, which include the Authority under which the committee has been constituted, details of qualification, etc of chairman and the members, procedures for replacement or removal of members, maintenance of records, Standard Operating Procedures (SOPs) to be followed by the committee for various activities like policy regarding training of members, prevention of conflict of interest, procedures 
for vulnerable population etc.

iii. The Ethics Committee shall review and accord its approval to a clinical trial and also carry ongoing review of the trial at appropriate intervals, as specified in Schedule Y and the Good Clinical Practice Guidelines for Clinical Trials in India and other applicable regulatory requirements for safeguarding the rights, safety and well-being of the trial participants.

iv. In the case of any serious adverse event occurring to the clinical trial participants during the clinical trial, the Ethics Committee shall analyse and forward its opinion as per procedures specified under Appendix XII of Schedule Y.

v. The Ethics Committee shall allow inspectors of officials authorized by the DCG (I) to inspect their facilities, records, documents etc.

vi. The registration of an Ethics Committee shall be valid for a period of three years from the date of issue.

vii. If the Ethics Committee fails to comply with any of the conditions of registration, DCG (I) may suspend or cancel the registration of the Ethics Committee.

\section{Future of clinical trial regulations in india}

\section{Accreditation of iec, investigators and sites}

Any clinical trial of a new or existing drug or medical device needs to be carried out at a centre where facilities for carrying out such trials exist, and the investigators carrying out the trial are competent and have experience in the successful conduct of such trials. In addition, the institute ethics committee (IEC) of the institute where the trial is to be carried out should understand the ethics involved in clinical trials and should consist of persons from different walks of life.

When a clinical trial is carried out under such conditions, it gives confidence to the volunteer and patients participating in the trial that they will not be subjected to any unjustified or hazardous trial. It also gives confidence to the drugs regulator and the government that the trial is going to be well-run, and a sense of confidence to the public and the civil society that the clinical trials being carried out are justifiable, both on ethical and scientific grounds.

To ensure implementation of these concepts, it is proposed to introduce a system of accreditation for the following:

a) Institute ethics committees (IECs) b) Principal investigators (PIs) of clinical trials

c) Centres carrying out clinical trials.

Clinical trials will only be allowed to be conducted at those centres which have been accredited in all three areas as above. These centres could be in the public or the private sector. Conducting clinical trials at centres that have not been accredited would be illegal and will not be recognized by the Drugs Controller General of India (DCGI). The accreditation process will be supervised by a Central Accreditation Council (CAC).

\section{Central Accreditation Council (CAC)}

This will be a semi-autonomous council consisting of three full-time members including the chairperson. They will be senior clinicians selected' on the basis of their competence, good track record, integrity and competence from different parts of the country, keeping gender sensitivity in mind. The Council will have full-time employees and its own secretarial staff. The Council will supervise the accreditation of all three areas, viz. IECs, PIs and centres carrying out clinical trials. The Council may ask agencies to carry out accreditation, or may do so themselves. The CAC needs to have an independent status and not be a subsidiary of the Central Drugs Standard Control Organization (CDSCO). The decisions of the CAC will be final.

\section{Specific recommendations}

- Accreditation will require to be done of centres carrying out clinical trials, PIs and IECs. Only centres with all three accreditations would be allowed to carry out clinical trials by the Drugs Controller General of India (DCGl).

- ACentral Accreditation Council(CAC) should be set up which will oversee the accreditation of these three based on the criteria adopted and keep the three registers listing the accredited entities/persons updated.

- Selection of assessors should be carried out by a blind randomization procedure.

- A course for becoming a certified clinical investigator should be set up so that the much-needed pool of clinicians eligible to become PIs can be created.

Report of the prof. Ranjit roy chaudhury expert committee

Overview of the Drugs and Cosmetics (Amendment) Bill, 2013: Regulations For Medical Devices and Conduct of 


\section{Clinical Trial.}

Drugs and Cosmetics (Amendment) Bill, 2013 that will soon be enacted would act like a boon to the industry and would bring along, positive hopes to achieve an assurance of the best and safest medical technologies in the country.

In 2006, the Government realized that the country requires a legislation to bring under its control the safety and performance of medical devices and accordingly introduced the Medical Devices Regulation Bill, 2006 with the intention to consolidate laws related to medical devices and establish the Medical Device Regulatory Authority of India (MDRA). This Bill was aimed at establishing and maintaining a national system of controls for the quality, safety and availability of medical devices in India. However, the above Bill has not been enacted by the Parliament.

At present, provisions related to import, manufacture, distribution and sale of medical devices are covered under the Drugs and Cosmetics Act, 1940 ["Act"] and the Drugs and Cosmetics Rules, 1945 ["Rules"]. However, the said Act and the Rules cover only notified Medical Devices2. Notified medical devices are those medical devices which have been notified as drugs by the Government of India. Notified Medical Devices are currently covered under the definition of Drugs under the Act under Section 3 (b) (iv) which reads as follow:

\section{"b) "Drug" includes}

(iv) such devices intended for internal or external use in the diagnosis, treatment, mitigation or prevention of disease or disorder in human beings or animals, as may be specified from time to time by the Central Government bynotification in the Official Gazette, after consultation with the Board"

The Drugs and Cosmetics (Amendment) Bill , 2013

The most recent measure taken up by the Government on 29 August, 2013 is the introduction of the Drugs and Cosmetics (Amendment) Bill, 2013. The Bill 2013 contains a separate Chapter for regulatory provisions for import, manufacture, sale, distribution and export of medical devices and for regulating conduct of clinical trials in India. This Bill 2013 also provides provisions for setting up of a Central Drugs Authority (CDA) as an overarching body for regulation of drugs and cosmetics. The Authority shall have power to issue a license or a certificate, as the case may be, for the manufacture for sale or for export of drugs specified in the Third Schedule to the Act.

\section{Regulations on the Medical Devices}

In terms of medical device, the Bill 2013 has thrown light on the definition of the term "medical device" on the same approach as that of MDRB. In the Bill 2013, medical device includes additionally the instruments for diagnosis, monitoring, treatment, alleviation of, or assistance for handicaps also. Once the Bill is passed, the definition of medical device will no longer be read in the light of the definition of "drugs" as specified under Section 3(b) (IV) of the Drugs and Cosmetics Act, 1940 which creates ambiguity and restricts the ambit of medical device only to notified medical devices.

Provisions related to import, manufacture, sale, distribution and export of medical devices

The import, manufacture, sale, distribution, export and labeling of notified medical device is regulated under the Drugs and Cosmetics Act, 1940. However, it is felt by the industry and regulatory authorities that provisions related to drugs cannot be applicable entirely for medical devices. In this context, the present Bill 2013 is an attempt to frame provisions which are specific for medical devices. For instance, the Bill 2013 specifies the conditions under which medical devices shall be deemed to be misbranded, adulterated, and spurious or not of standard quality. In order to define when a medical device shall be deemed to be misbranded under Section $7 \mathrm{C}$ of the Bill, it has made new insertion of the term "functional value" and states as "...........if it is made to appear of better or greater therapeutic or functional value than it really is..." Similarly, a new sub-clause (e) has been inserted in Section 7D of the Bill 2013 which defines when a medical device shall be deemed to be adulterated drugs and the same reads as "if its containers is composed, in whole or in part, of any deleterious substance which may render it dangerous to use or injurious to health."

The Bill 2013 restricts any person to import or manufacture for sale or for export by himself or by any person on his behalf those medical devices which are not of standard quality or misbranded, adulterated, spurious under Section $7 \mathrm{~F}$ and it has also inserted a new sub-clause that includes restriction for import or manufacture for sale or for export any software or part or component or instrument or the list of the software or part or ingredient or instrument contained in it, unless displayed in the prescribed manner on 
the label or container.

The Bill 2013 has also laid down the penalties for the offences related to import, manufacture for sale, stocking, exhibiting, offering for sale of medical devices or distribution or export of any adulterated, spurious or not of standard quality medical devices and penalties related to grievous injury or death caused due to use of such medical devices. Further, Section $7 \mathrm{M}$ specifies provisions for confiscation of medical device, implements, machinery, receptacles, packages, coverings, animals, vehicles, vessels or other conveyances of persons convicted for offences under Chapter II A of the Bill 2013.

\section{Advisory body pertaining to the matters related to the medical devices}

The Bill 2013 includes Section 5A which specifies the establishment of a Medical Devices Technical Advisory Board which will act as an advisory body to the Central Government, the Central Drugs Authority and State Governments on technical matters pertaining to medical devices, arising out of the administration of the Bill 2013 and to carry out other functions assigned to it by or under the purview of the Bill 2013.

Similarly, another advisory committee called "the Drugs, cosmetics and Medical Devices Consultative Committee" may also be constituted by the Central Government which would require to provide advice to the Central Government, the Central Drugs Authority and State Governments on matters pertaining to secure uniformity throughout India in the administration of the provisions of the Bill 2013.

BILL NO. LVIII OF 2013 A Bill further to amend the Drugs and Cosmetics Act, 1940

Formation of the Central Drugs Authority (CDA)

Further, the Bill 2013 has inserted Chapter I A that specifies the structurization of new regulatory authority- the Central Drugs Authority. The CDA will be empowered to act like a regulatory check on the functioning of the Central Licensing Authority and the State Licensing Authorities. In fact, in order to attempt for diligent functioning of these authorities, CDA can assess periodically the functioning of the authorities and further specify regulations, guidelines, norms, structures and requirement for effective functioning. In the recent years, there have been several reports of irregularities in issuance of permissions, licenses or certificates and permits by the State authorities. Keeping in view such circumstances, the Bill 2013 empowers the CDA to review, suspend or cancel any permission, license or certificate issued by the Central Licensing Authority or the State Licensing Authorities and it extends to issue, renew, suspend or cancel such license, certificate, approvals and permissions for conducting clinical trial as well.

\section{The Central Drugs Authority shall}

a) Specify, by regulations, the guidelines, norms, structures and requirements for effective functioning of the Central Licensing Authority and the State Licensing Authorities;

b) Assess periodically the functioning of the Central Licensing Authority and the State Licensing Authorities;

c) Have power to issue directions to the Central Licensing Authority and the State Licensing Authorities to ensure compliance with the guidelines, norms, structures and requirements specified by it under clause (a);

d) Review, suspend or cancel any permission, licence or certificate issued by the Central Licensing Authority or the State Licensing Authorities;

e) Specify, by regulations, the fees or charges for issue or renewal of licences, certificates, approvals and permissions by the Central Licensing Authority and the State Licensing authorities;

f) Coordinate, mediate and decide upon the disputes arising out of the implementation of the provisions of the Act and rules and regulations made there under between two or more States Licensing Authorities;

g) Constitute such committees or sub-committees as it considers necessary for the efficient discharge of its functions and exercise of its powers under this Act;

h) Recommend to the Central Government the measures as regards the standards of drugs, cosmetics and medical devices for effective implementation of the provisions of this Act;

i) Perform such other functions as may be prescribed by the Central Government.

\section{Regulations with respect to conduct of clinical trial in the Bill 2013}

The insertion of Chapter IB on the provisions related to clinical trial will strengthen statutory protections related to the serious risk involved in the conduct of clinical trial. A new insertion of provision specifies that the Central 
Licensing Authority may, in public interest, abbreviate, defer or omit the pre-clinical and clinical data requirements for approval of clinical trial of drugs indicated in life threatening or serious diseases or diseases of special relevance to the country. Further, Bill 2013 has inserted provisions related to stringent punishment for the companies conducting clinical trial without permission or in contravention of any provisions specified in the Bill 2013 and provisions related to compensation for adverse effects of clinical trial suffered by the participants of clinical trial. The Bill 2013 also lays down that the companies which are held responsible to pay compensation to the victims of clinical trial who suffered injury or death as a result of the adverse effect of the clinical trial companies, fails to do so, shall be punished with imprisonment which may extend to two years and with fine which shall not be less than twice the amount of the compensation.

The Bill 2013, once passed, will govern import, export, manufacture, distribution and sale of the medical devices and conduct of the clinical trials in India. Further, the Bill 2013 would completely overhaul all existing standards and lead to systematize the activities in the market related to medical devices and consequently, it will attract more international medical device companies to show willingness to manufacture products locally or import medical devices because of their assured quality and standard. Further, provisions relating to conduct of clinical trials which have been addressed extensively are a significant step towards implementation of Good Clinical Practice- a practice appreciated worldwide.

\section{BILL NO. LVIII OF 2013 A Bill further to amend the} Drugs and Cosmetics Act, 1940

\section{A. Transparent And Equitable System Of Clinical Evaluation Of New Drugs}

The system of clinical evaluation of new drugs that is being proposed will be ethical, transparent and equitable. The proposed system will be based on recommendations and decisions taken solely on a scientific basis. Once modifications suggested in this report have been implemented, there will be little opportunity of introducing bias or of a nexus developing in the system.

\section{Control mechanisms}

The modified system will depend on the following mechanisms to make it efficient, transparent and equitable:

1. Accreditation of all centres carrying out clinical trials, principal investigators and institute ethics committees

2. Standard operating procedures (SOPs) to be followed for all steps

3. Randomization of the system of allocations of experts for review of applications and Assessment of centres for accreditation

4. Use of information technology at every stage to ensure transparency of the system

5. Use of a single window system

6. A process of dialogue to be set up for communications

7. Establishment of a computerized database of carefully selected experts, which will be updated every year.

Report of the prof. Ranjit roy chaudhury expert committee

\section{Steps of the trial (Single Window system)}

Pharmaceutical house or investigator will be given an acknowledgement and a reference number by CDSCO. Check status Online. Applicant to be given a written assurance that if all the papers needed for the review are complete, then a decision, either interim or full, will be given within three months.

\section{$\downarrow$}

Application will be reviewed by the Technical Review Committee (TRC)

\section{$\downarrow$}

Once the TRC approves the clinical trial proposal the sponsor is informed of the same

Proposal will be sent to the Randomization Unit (which may or may not be situated at the CDSCO). This unit will list at least six accredited centres suitable for carrying out trials with the drug.

\section{$\downarrow$}

The sponsor/applicant will be required to select two or three centres, as recommended by the TRC. The Chief Investigator, the heads of the centres and the $\mathrm{CDSCO}$ will be informed of the centres selected.

Proposal with the protocol will be submitted to the Institute Ethics Committee (IEC) for ethical review and approval. 
If any modification to the protocol is introduced by the Principal Investigator, the CDSCO will need to be informed. If the modification is a major one, it will need to be approved by the TRC.

\section{$\downarrow$}

After IEC approval, this information will be entered into the central trial registry

\section{$\downarrow$}

The clinical trial will be initiated.

\section{$\downarrow$}

Progress of the clinical trial will be reported every six months to the IEC and the CDSCO.

\section{$\downarrow$}

Serious adverse events (SAEs) during the trial, if any, will be reported to the IEC.

\section{$\downarrow$}

The final report of the clinical trial will be submitted to the CDSCO and the IEC.

\section{$\downarrow$}

The final report will be reviewed by the TRC of the CDSCO, and if approved, the CDSCO will inform the sponsor/applicant of the outcome of the trial.

The results of the clinical trial, the approval of the IEC and the final decision of the TRC will be placed on the website of the CDSCO.

\section{$\downarrow$}

CDSCO will take the subsequent steps regarding the drug based on the recommendations of the Technical Advisory Committee.

Report of the prof. Ranjit roy chaudhury expert committee

Actions on the Recommendations of Prof. Ranjit Roy Chaudhury Expert Committee

Ministry of Health \& Family Welfare had constituted an Expert Committee under the Chairmanship of Prof. Ranjit Roy Chaudhary to formulate policy and guidelines for approval of new drugs, clinical trials and banning of drugs. The actions proposed to be taken on the recommendations of the Expert Committee, as finalized by the Ministry of Health \& Family Welfare after discussion with the Committee members are given below:

\section{Clinical Evaluation of New Drugs}

a) Accreditation of Ethics Committee, Investigators and the clinical trial sites.

b) Procedure for review of applications of clinical trials and new drugs. New Drug Advisory Committees (NDACs) will be renamed as Subject Expert Committees. Applications of clinical trials and new drugs will initially be evaluated by the Subject Expert Committees and their recommendations will be reviewed by the Technical Review Committee (TRC). CDSCO will grant approval of Clinical Trial and New Drugs based on the recommendations of TRC.

c) Computerized database will be created and selection of experts will be based on specific criteria.

d) Requirement of filing application to market New Chemical Entities (NCEs) if India participated in Global clinical trials of those NCEs.

e) Specifying time line of six months for disposal of applications for approval of clinical trials and new drugs for processing of applications

f) All proposals need not be evaluated by Technical Review Committee (TRC)

g) The pharmaceutical companies, the Investigators, the drugs regulator and the ECs all would have to ensure that the design used in a placebo controlled clinical trial is appropriate, efficient and ethical.

h) Post trial access of investigational product to the trial participants.

i) Audiovisual recording of the informed consent process is mandatory.

j) Action in case of violation of the informed consent processes.

k) Number of clinical trials an Investigator can undertake at a time should not be more than three at a time.

1) Use of Information technology at all steps of a clinical rial to ensure total transparency in the system.

m) Monitoring of clinical trials.

n) All NCEs/NMEs undergoing clinical trials anywhere can undergo parallel Phase II and Phase III Clinical trials in India after carrying out safety assessment of Phase I Clinical Trial data generated abroad.

o) Clinical Trial of Medical Device is different in nature as compared to that of Drugs or Vaccine. In case of 
Medical Device, there is no concept of conducting Phase I Clinical trial to assess safety, tolerability of the Medical Device.

p) Reporting of Serious Adverse Events (SAEs) by the Investigator within $24 \mathrm{hrs}$ and in case the investigator fails to report any serious adverse event within the stipulated period, he shall have to furnish the reason for the delay to the satisfaction of the DCG(I) along with the report of the serious adverse event.

q) Compensation in case of injury or death due to failure of intended therapeutic effect of investigational product.

r) Medical management in case of serious adverse events.

s) Compensation in case of injury or death due to use of placebo in place of standard therapy.

t) Compensation in case of clinical trial related injury or death and Compensation need not be paid for injury or death due to totally proven unrelated causes.

u) Compensation for injury or death due to SAE caused by a procedure undertaken to deal with an SAE caused by the original drug being evaluated.

v) Compensation in case of injury or death discerned at a later stage should be paid to the trial participant.

w) Ancillary care to patients suffering from any other illness during the trial.

x) Academic clinical research may be approved by the Institutional Ethics Committee (IEC).

y) Compensation in case of injury or death in academic trial.

z) Compensation in case there is an increase in the number of SAEs in clinical trials being carried out on patients suffering from terminal illnesses.

aa) Causality analysis of SAEs to determine the cause of injury or death.

\section{Approval of New Drugs}

a) There should not be any fixed number of drugs / drug trials that should be approved in the country.

b) Deletion of existing drugs, if a new drug is approved.

c) Requirement for a new drug to have advantage over the existing drug for considering approval of the new drug.

d) Requirement of conduct of all phases from Phase I to Phase IV trial in the country for new entities developed in India.

e) Waiver of Clinical Trial in Indian population for approval of new drugs, which have already been approved outside.

f) Consideration of ethnicity for approval of new drugs.

g) Approval of new drugs already approved in other countries, based on clinical trial conducted in Indian patients as part of global clinical trial

h) Requirement of clinical trial for a drug which is considered generic drug in other country like USA but not approved in India

i) Requirements of local trial for a generics or similar biologics (Biosimilars) in other country like USA for its approval in the country

j) Requirement of Bioequivalence (BE) study for subsequent approval of new drugs already approved in the country

k) Similar biologics (Biosimilars) would require both preclinical development and bridging Phase III clinical trials as per Department of Biotechnology (DBT) CDSCO guidelines for its approval in India.

1. Post Marketing Surveillance

Post Marketing Surveillance for six years

Reporting of Adverse Drug Reactions (ADR) of marketed drugs by the pharmaceutical company, clinician treating the patient or his/her hospital, or even a practicing clinician

a) Restriction of use of new drug for a year or two only in hospitals

b) Continued permitting of Bioavailability / Bioequivalence (BA/BE) studies for export purpose

2. Banning of drug

a) Weeding out of hazardous / irrational drugs from the market

b) Consideration of banning of a marketed drug if two or more countries remove the drug from their market due to issues related to safety and efficacy of the drug.

c) Continued evaluation of drugs marketed in the country

3. Strengthening of CDSCO

a. Overall strengthening and up gradation of CDSCO

b. Creation of a research unit within the CDSCO

c. Interaction with the applicants at any point of time

d. Rank and status of DCG(I) to be upgraded

e. Strengthening of CDSCO in terms of manpower 
f. Updating skill and knowledge of regulators and experts

g. Effective communication system of CDSCO

h. Licensing of manufacture of drugs by State Licensing Authority / Central Licensing Authority. In the Drugs and Cosmetics (Amendment) Bill, 2013, it has been proposed to have Central Licensing for 17 critical categories of drugs.

i. Strengthening of Pharmacovigilance programme http://cdsco.nic.in/Action_RR_Choudhury _Committee_06.11.2013

\section{REFRENCES}

1. Sharma H, Parekh S (2012) Clinical Trials Regulations in India. Pharmaceut Reg Affairs 1:e118. doi:10.4172/2167-7689.1000e118

2. Imran M, Najmi AK, Rashid MF, Tabrez S, Shah MA. Clinical research regulation in India-history, development, initiatives, challenges and controversies: Still long way to go. J Pharm Bioall Sci 2013;5:2-9.
3. Carrying out Clinical Trials in India Jayasheel B G www.rajpharma.com RAJ Pharma June 2010

4. Recent Changes in Regulations Related to Clinical Trials Approval in India Dr. Jayasheel B. G. Pharma Times - Vol. 44 - No. 05 - May 2012

5. Suprabha Naralkar, Kishor Karande,Pratik Vasani, Pharmalink ConsultingPvt. Ltd, India;Regulatory Rapporteur Vol 7, No 2, February 2010

6. CDSCO and Ministry of health and welfare website 